\title{
Controllable nonlocal transport of Majorana fermions with the aid of two quantum dots
}

\author{
Jie Liu, Jian Wang, and Fu-Chun Zhang \\ Department of Physics, and Center of Theoretical and Computational Physics, The University of Hong Kong, Hong Kong, China
}

(Received 24 June 2013; revised manuscript received 16 June 2014; published 14 July 2014)

\begin{abstract}
Majorana fermions (MFs) are appearing in pairs and nonlocally distributed at the two ends of the wire. The two MFs can combine together to form a nonlocal fermion state. Due to the nonlocal nature of Majorana fermions in the topological superconductor, there are two types of nonlocal transport processes supported by MFs: crossed Andreev reflection and electron transmission. In this paper, we study electron transport through a normal lead-quantum dot-topological superconductor-quantum dot-normal lead (N-QD-TS-QD-N) junction. We show that these two nonlocal processes can be directly controlled by gating the energy level of QDs. For instance, when both energies of QDs (labeled as $\epsilon_{1}$ and $\epsilon_{2}$ ) are equal to the coupling energy of MFs (denoted as $E_{M}$ ), the electron in the left lead can transport to the right lead via MFs while when $\epsilon_{1}=-\epsilon_{2}=E_{M}$ is satisfied, the electron in the left lead can combine one electron in the right lead to form a Cooper pair and tunnel into the topological superconductor via MFs.
\end{abstract}

DOI: 10.1103/PhysRevB.90.035307

PACS number(s): 74.78.Na, 74.25.F-, 74.45.+c, 74.81.Fa

\section{INTRODUCTION}

Majorana fermions (MFs) are a special type of particles which are their own antiparticles and obey novel non-Abelian statistics [1,2]. Due to their fundamental importance and potential application in topological quantum computing, they have attracted great attention to seek the realization of MFs in solid-state systems. Indeed, a series of proposals to search for MFs in condensed matter systems have been put forward [3-12]. One of the promising proposals is that realizing zero-energy states in one-dimensional (1D) superconducting wires by inducing superconductivity on semiconductor wires with Rashba spin-orbit coupling [6-8]. Due to the particle-hole symmetry of superconductor, these zero-energy states are selfHermitian and should be MFs. When tunneling from a norm wire into a semiconductor superconducting wire, these zeroenergy states can cause a quantized $G(0)=\frac{2 e^{2}}{h}$ conductance peak at zero bias $[13,14]$. This transport property is suggested as a simplest way to directly detect MFs. Soon after this proposal, several groups have fabricated the semiconductor superconducting wire and observed zero-bias peaks (ZBP), indicating the existence of MFs [15-17]. These observations have made an important first step towards the realization of MFs in solid-state systems. However, the interpretation of the ZBP is not unique. An ordinary localized state could also give rise to a $\mathrm{ZBP}$, and it is difficult to distinguish whether these observed ZBPs are induced by MFs or ordinary localized states [18-25]. Therefore, it remains highly controversial as to whether the ZBP has captured the signature of MFs and is necessary to further study the novel transport properties of MFs.

Aside from the self-Hermitian of MFs, another main feature of MFs is that MFs are nonlocally distributed at two ends of the wire [3]. The origin of nonlocality of MFs is due to the fact that a MF can be viewed as half of an ordinary fermion. To define a quantum state with MFs we must consider a pair of nonlocally distributed MFs $\gamma_{1}$ and $\gamma_{2}$. They combine together to form a nonlocal fermionic state via the relation $f=\gamma_{1}+i \gamma_{2}$, where $f$ is an ordinary fermion operator. The nonlocal nature of MFs can cause two novel nonlocal transport phenomena: the crossed Andreev reflection (CAR) illustrated in Fig. 1(c) and the electron transmission (ET) in Fig. 1(d) [26,27]. In the CAR process, an electron tunnels into the superconductor from one lead and then tunnels out as a hole at the other lead, while in the ET process an electron tunnels out as an electron instead of a hole at the other lead. While the AR is a local transport process in which an electron tunnels into a superconductor with a hole being reflected back from the same lead as illustrated in Fig. 1(b). In general, all three processes can occur and mix together. In our previous paper, we suggest that the nonlocal correlation is a good indicator to distinguish these processes [28]. However, it will be more interesting to manipulate, control, and measure these nonlocal processes directly.

In this work, we use two QDs to confine a topological superconductor to form a normal lead-QD-topological superconductor-QD-normal lead (N-QD-TS-QD-N) junction. The experimental setup is depicted in Fig. 1(a). Some of the physics in such a junction has been studied in several papers [29-32]. These papers do reveal many exotic properties of MFs, such as the nonlocal correlation of MFs, with the aid of QDs. Here, we focus on how to manipulate and control the nonlocal transport directly. It may have giant potential for further application. Our calculations show that strongly coupled MFs can be confined well within the topological superconductor provided that the QDs are weakly coupled to the MFs and the energy level of QDs are tuned away from the Fermi energy of superconductor. In this situation, the local $\mathrm{AR}$ is largely suppressed and the nonlocal tunneling event is dominant. In addition, these nonlocal transport processes can be directly controlled through gating the energy level of QDs. When the energy levels of both QDs (labeled as $\epsilon_{1}$ and $\epsilon_{2}$, respectively) are equal to the coupling energy of MFs (denoted as $E_{M}$ ), an electron in the left lead can be perfectly transmitted to the right lead via MFs. When the energy level of one QD $\epsilon_{1}$ equals to $E_{M}$ while $\epsilon_{2}$ equals to $-E_{M}$, an electron in the left lead may combine with the second electron in the right lead to form a Cooper pair and resonantly tunnel into the topological superconductor via MFs. These controllable nonlocal transport properties directly manifest the nonlocal nature of MFs. 

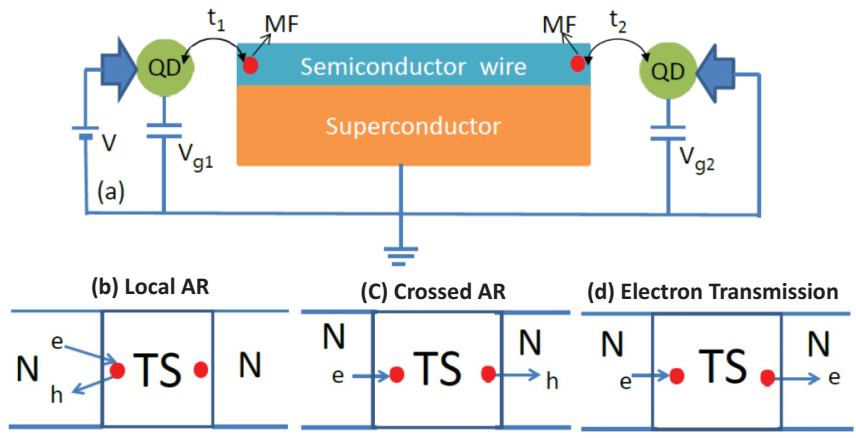

FIG. 1. (Color online) (a) The schematic plot of experimental setup: it can be used to detect the nonlocality of MFs. (b) Local Andreev reflection process: an electron from one lead is reflected as a hole in the same lead. (c) Crossed Andreev reflection: an electron from one lead tunnels to the other lead as a hole and a Cooper pair is injected into the superconductor. (d) Electron transmission: an electron from one lead transmit to the other lead via MFs.

\section{MODEL AND FORMALISM}

The model is described as Fig. 1(a): two QDs are connected at the two ends of the topological superconducting wire. When the chemical potential of superconducting wire lies in the topological region, two MFs will appear at the two ends of the wire, respectively. In the low-energy case, the effective Hamiltonian of QD-TS-QD system can be written as follows [27,29]:

$$
\begin{aligned}
H_{0}= & \sum_{\mathbf{i}} \varepsilon_{\mathbf{i}} \mathbf{d}_{\mathbf{i}}^{\dagger} \mathbf{d}_{\mathbf{i}}+i \mathbf{E}_{\mathbf{M}} \gamma_{1} \gamma_{2}+\mathbf{t}_{1}\left(\mathbf{d}_{1}^{\dagger}-\mathbf{d}_{1}\right) \gamma_{1} \\
& +i \mathbf{t}_{2} \gamma_{2}\left(\mathbf{d}_{2}^{\dagger}+\mathbf{d}_{2}\right),
\end{aligned}
$$

where $\gamma_{1}$ and $\gamma_{2}$ are the Majorana operators and the parameter $\mathbf{E}_{\mathbf{M}} \propto e^{-l / \xi_{0}}$ describes the coupling energy between the two MFs [26,33], $l$ is the length of wire, and $\xi_{0}$ is the superconducting coherence length. Here, $\mathbf{d}_{1}$ and $\mathbf{d}_{2}$ are the annihilation operators in the left and right $\mathrm{QD}$, respectively, and $\mathbf{t}_{1}\left(\mathbf{t}_{2}\right)$ represents the coupling strength between the left (right) QD and the MF $\gamma_{1}\left(\gamma_{2}\right)$.

Since it is convenient to work in the conventional fermion representation, we can transform the Hamiltonian (1) to the following form using relations $\gamma_{1}=f+f^{\dagger}$ and $\gamma_{2}=$ $-i\left(f-f^{\dagger}\right)$ :

$$
\begin{aligned}
\tilde{H}_{0} & =H_{e}+H_{e h}, \\
H_{e} & =\sum_{\mathbf{i}} \varepsilon_{\mathbf{i}} \mathbf{d}_{\mathbf{i}}^{\dagger} \mathbf{d}_{\mathbf{i}}+\mathbf{E}_{\mathbf{M}} f^{\dagger} f+\left(\mathbf{t}_{1} \mathbf{d}_{1}^{\dagger} f+\mathbf{t}_{2} f \mathbf{d}_{2}^{\dagger}+\text { H.c. }\right), \\
H_{e h} & =\mathbf{t}_{1}\left(\mathbf{d}_{1}^{\dagger} f^{\dagger}-\mathbf{d}_{1} f\right)+\mathbf{t}_{2}\left(f \mathbf{d}_{2}-f^{\dagger} \mathbf{d}_{2}^{\dagger}\right),
\end{aligned}
$$

where the second term $H_{e h}$ is the anomalous Hamiltonian which converts two electrons into a Cooper pair. To study the transport properties of such a system, each QD is connected to a lead. Assuming the tunneling rate of the lead is energy independent, the Green's function of the system is easily calculated in the Nambu representation [34,35]

$$
G^{r}=\left[E-\left(\begin{array}{cc}
\tilde{H}_{e}+\Sigma_{e}^{r} & \tilde{H}_{e h} \\
\tilde{H}_{e h}^{\dagger} & -\tilde{H}_{e}^{*}+\Sigma_{h}^{r}
\end{array}\right)\right]^{-1},
$$

where $\tilde{H}_{e}$ is the matrix representation of $H_{e}$ with the basis $\left(d_{1}^{\dagger}|0\rangle, f^{\dagger}|0\rangle, d_{2}^{\dagger}|0\rangle\right)^{T}, \tilde{H}_{e h}$ is the matrix representation of $H_{e h}$, $\Sigma_{e}^{r}=\Sigma_{h}^{r}=\operatorname{diag}\left(-i \Gamma_{L} / 2,0,-i \Gamma_{R} / 2\right)$ is the self-energy due to the leads, and $\Gamma_{L(R)}$ is the linewidth function of the left (right) leads. We can calculate the current from the left (right) lead directly [35]:

$$
\begin{gathered}
I_{L}=I_{L A}+I_{L e}+I_{L h}, \\
I_{L A}=\frac{e}{h} \int d E \operatorname{Tr}\left[\Gamma_{L} G_{14}^{r} \Gamma_{L} G_{41}^{a}\right]\left(f_{L e}-f_{L h}\right), \\
I_{L e}=\frac{e}{h} \int d E \operatorname{Tr}\left[\Gamma_{L} G_{13}^{r} \Gamma_{R} G_{31}^{a}\right]\left(f_{L e}-f_{R e}\right), \\
I_{L h}=\frac{e}{h} \int d E \operatorname{Tr}\left[\Gamma_{L} G_{16}^{r} \Gamma_{R} G_{61}^{a}\right]\left(f_{L e}-f_{R h}\right),
\end{gathered}
$$

where $G_{i j}^{r}$ is the matrix element of $G^{r}$ and $G_{j i}^{a}=\left(G_{i j}^{r}\right)^{\dagger}$ and the index $i=1$ (4) labels the electron (hole) of the left QD while $i=3$ (6) labels the the electron (hole) of the right QD. $f_{L(R) e(h)}$ is the electron (hole)'s Fermi function in the left (right) lead. The physical meaning of the current is obvious: $I_{L A}$ is the current in the left lead due to the local AR with the transmission coefficient $T_{A}=\operatorname{Tr}\left[\Gamma_{L} G_{14}^{r} \Gamma_{L} G_{41}^{a}\right]$, while $I_{L e}$ is the current which is contributed by the electron teleportation process with the transmission coefficient $T_{e}=\operatorname{Tr}\left[\Gamma_{L} G_{13}^{r} \Gamma_{R} G_{31}^{a}\right]$, and $I_{L h}$ is the current from the contribution of CAR with the transmission coefficient $T_{h}=\operatorname{Tr}\left[\Gamma_{L} G_{16}^{r} \Gamma_{R} G_{61}^{a}\right]$.

\section{RESULTS AND DISCUSSION}

In the following, we will study the transport properties of the QD-TS-QD system. As discussed in our previous paper $[18,28]$, we set the superconducting gap $\Delta$ as our energy unit. The coupling strength of MFs can be on the order of 0.1 and $\Gamma_{L / R}$ can be on the order of 0.01 . Hence, we set $\Gamma_{L}=\Gamma_{R}=0.03$ in the following calculation. First, we consider the case that the QDs are strongly coupled with the MFs with $t_{1}=t_{2}=0.1 \sim E_{M}$. Figure 2(a) shows the AR coefficient $T_{A}$, the CAR coefficient $T_{h}$, and the ET coefficient $T_{e}$ as a function of incident energy $E$ where the energy levels of two QDs $\varepsilon_{1}=\varepsilon_{2}=0$ are in line with the Fermi level (which is set at superconducting condensate) and the coupling energy of MFs $E_{M}$ is set to zero (in the case of a long superconducting wire). In this case, only the local AR can occur while the nonlocal processes such as the CAR and ET are prohibited, which is understandable since zero coupling energy $\left(E_{M}\right)$ means no communication between two MFs. When $E_{M}$ is nonzero, the nonlocal processes are allowed. Figure 2(b) depicts the results when $E_{M}=0.2$ with all other parameters kept the same as in Fig. 2(a). We see that there are four resonant peaks in $T_{e}$ and $T_{h}$ as the incident energy $E$ is varied. In addition, $T_{A}$ also shows four peaks at the same energies. Thus, all three processes mix together and it is hard to distinguish them. Interestingly, $T_{A}$ also shows a zero-bias peak although the energies of MFs are no longer kept at zero due to the coupling between MFs. This is because there is an exactly zero-energy MF formed in the QD when the QD's energy level is in line with the Fermi level [36]. 

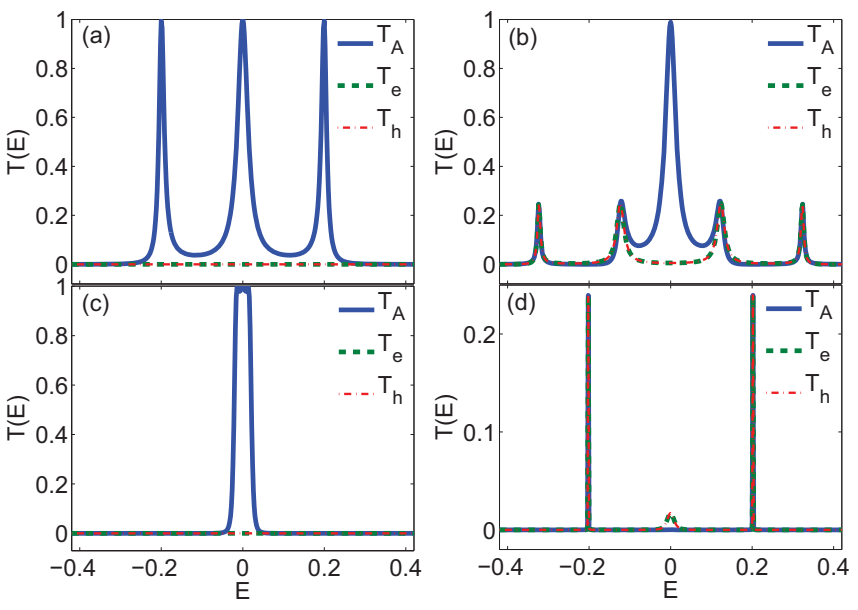

FIG. 2. (Color online) The transmission coefficients vs incident energy $E$ in various situations. (a) $E_{M}=0$ : in this case two MFs are well separated and only the local AR can occur. (b) $E_{M}=0.2$ : in this case, two MFs are coupled to each other and the nonlocal process can happen. In both (a) and (b), the MFs are well coupled with the QDs with $t_{1}=t_{2}=0.1$. (c) $E_{M}=0$ : the local AR still survives though $t_{1}=t_{2}=0.01$. (d) For $E_{M}=0.2$ and $t_{1}=t_{2}=0.01$, all the processes are suppressed due to the weak coupling between QDs and MFs except at $E= \pm E_{M}$. The energies of QDs are in line with the Fermi level in all the cases: $\varepsilon_{1}=\varepsilon_{2}=0$.

From Figs. 2(a) and 2(b) we can see that the system indeed behaves like a covalent molecule in the strongly coupling regime. All three processes, the local AR, CAR, and ET, can occur and mix together when $E_{M} \neq 0$. To observe the nonlocal processes such as the CAR and ET, we have to suppress the local AR process. A simple way to suppress the local AR is to decrease the coupling strength between MFs and QD. It is known that when the coupling strength between QD and a superconductor is much smaller than the superconducting gap, the local AR can be suppressed and only the nonlocal tunneling events are present [37]. Thus, with the decreasing of coupling strength between QDs and MFs, the local AR is largely suppressed. Figures 2(c) and 2(d) show the transmission coefficients versus the incident energy $E$ in the weak coupling regime with $t_{1}=t_{2}=0.01 \ll E_{M}$. In Fig. 2(c), $E_{M}=0$, we can see that the local AR still dominates and the tunneling probabilities of the nonlocal processes are zero. This is consistent with the previous result that the zero-bias peak would remain at the integer value $2 e^{2} / h$ regardless of the coupling strength between the leads and MFs when $E_{M}$ is zero. When $E_{M}$ is not strictly zero, however, the local AR is suppressed. In Fig. 2(d), $E_{M}=0.2$, we can see that all three processes are almost suppressed.

The most interesting thing is that by tuning the energy level of the QD in the weak coupling regime, we can completely suppress the local AR while allowing the processes of CAR and ET. In Figs. 3(a) and 3(b), we show the contour plot of $T_{h}$ and $T_{e}$, respectively, as functions of the right QD's energy $\varepsilon_{2}$ and the incident energy $E$. We have set the coupling energy of MFs $E_{M}=0.2$, the left QD's energy $\varepsilon_{1}=E_{M}$, and the coupling strength between QDs and MFs $t_{1}=t_{2}=0.01$. We did not show the contour plot of $T_{A}$ because it is almost zero as we
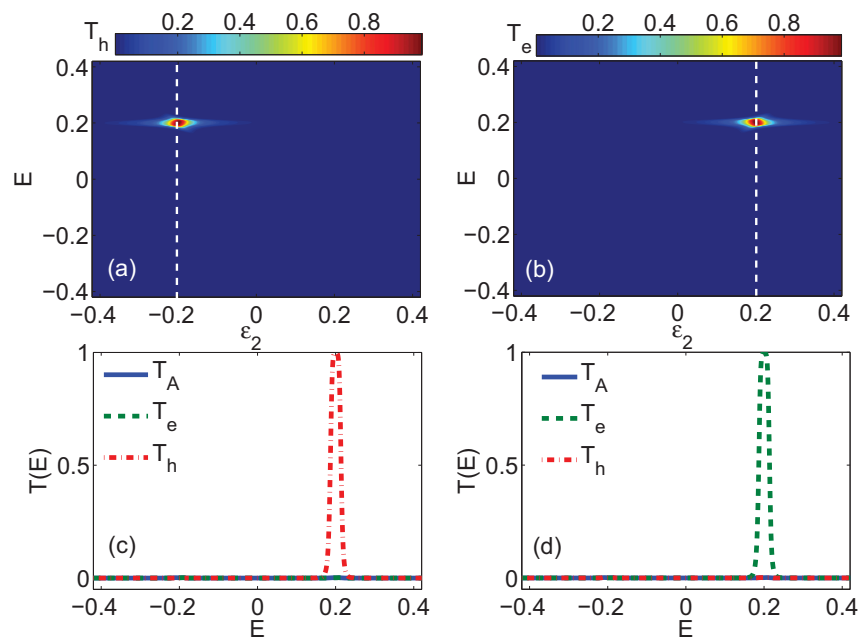

FIG. 3. (Color online) Contour plot of (a) $T_{h}$ and (b) $T_{e}$, as functions of right QD's energy level $\varepsilon_{2}$ and incident energy E. In both cases, $t_{1}=t_{2}=0.01$, and $\varepsilon_{1}=E_{M}=0.2$. (c) $T_{A}, T_{e}, T_{h}$ as a function of incident energy $E$ with the QD's energies fixed at $\varepsilon_{1}=-\varepsilon_{2}=E_{M}=0.2$ denoted by the dashed lines in (a). In this case, only the CAR survives. (d) $T_{A}, T_{e}, T_{h}$ as a function of incident energy $E$ with the QD's energy levels fixed at $\varepsilon_{1}=\varepsilon_{2}=E_{M}=0.2$ denoted by the dashed lines in (b). The ET is in the resonant regime.

demonstrated before. It is very interesting that there is a peak pinned at the location $\varepsilon_{1}=-\varepsilon_{2}=E_{M}=0.2$ in the contour plot of $T_{h}$ while no such a peak exists at the corresponding location in the contour plot of $T_{e}$. This means that only the CAR is allowed at this location while other processes are completely suppressed. Similarly, the contour plot of $T_{e}$ also shows a peak at the location $\varepsilon_{1}=\varepsilon_{2}=E_{M}=0.2$ indicating that the electron transmits from the left $Q D$ to the right $Q D$ via MFs via resonant tunneling while the other processes are suppressed. As we have known, the origin of ZBPs is not unique. It has been pointed out that ZBPs in topological superconductors can be caused by disorder-induced Andreev bounded states [18], weak antilocalization effect [20], and reflectionless tunneling effect [21]. However, all these effects are essentially happening at the interface between a normal lead and a superconductor. Due to this local nature, they can only support the local AR process and would not influence the results of the nonlocal transport of MFs. What is more, the local AR caused by these effects can naturally be suppressed when the QDs are tuning away from the Fermi surface. This is because the fact that here QDs only allow electrons with resonant energy $E=\varepsilon_{i}$ to pass the QDs. Thus, the local AR is naturally suppressed when the QDs are tuning away from the Fermi surface due to the fact that the Cooper pair should have zero energy.

In Fig. 3, we demonstrate the switching between two nonlocal transport processes by tuning the energy levels of QDs. It seems that it also can be realized in a trivial superconductor system with such a QD-superconductor-QD junction without need of MFs. However, in trivial superconductors these processes can only happen due to evanescent states that decay exponentially with the distance of the TS wire, making their probability extremely small for a long TS wire. This probability is usually on the order of 0.01 even if the distance 
of the TS wire is smaller than the coherence length [37]. As to the QD-TS-QD system, these processes happen with the help of two MFs which are nonlocally distributed at the two ends of the wire. Since two MFs can be seen as one state, an electron tunneling into the left end of the wire can appear at the right end with probability equal to one. This means that the resonant CAR and ET processes in the QD-TS-QD system can be realized. To emphasize these resonant processes, Fig. 3(c) shows the curves of $T_{A}, T_{h}, T_{e}$ as a function of incident energy $E$ while fixing $\varepsilon_{2}=-0.2$ [see the white dashed line in Fig. 3(a)]. We have also shown in Fig. 3(d) the curves of $T_{A}, T_{h}, T_{e}$ versus incident energy $E$ with $\varepsilon_{2}=0.2$ [the white dashed line in Fig. 3(b)]. We can see clearly that two resonant tunnelings occur (the peak height of these processes is 1): resonant $\mathrm{CAR}$ at $\varepsilon_{1}=-\varepsilon_{2}=E_{M}=0.2$ and resonant ET at $\varepsilon_{1}=\varepsilon_{2}=E_{M}=0.2$. Furthermore, the peak height will not decrease with the increasing of wire length as long as the condition $t_{1}=t_{2}=0.01 \ll E_{M}$ is satisfied. This demonstrates again the nonlocal nature of MFs.

Here, we emphasize that the coupling energy of MFs $E_{M}$ plays an important role in these processes. Due to the coupling between MFs and QDs, energy levels of MFs broaden with a width of order $t_{1}$ or $t_{2}$. If $E_{M}$ is much larger than $t_{1}$ (the coupling strength between MFs and QDs), then the occupied state and the unoccupied state can be distinguished. In other words, there is no mixing between ET and CAR processes. Figures 4(a) and 4(b) show the contour plots of $T_{h}$ and $T_{e}$, respectively, as functions of $\mathrm{E}$ and $E_{M}$ where we set $\varepsilon_{1}=\varepsilon_{2}=E_{M}$ and $t_{1}=t_{2}=0.01$. While Figs. 4(c) and 4(d) show the contour plots of $T_{h}$ and $T_{e}$, respectively, as functions of $\mathrm{E}$ and $E_{M}$ with $\varepsilon_{1}=-\varepsilon_{2}=E_{M}$. It clearly shows that two processes ET and CAR can occur at the same time when $E_{M} \sim t_{1}$ or $t_{2}$ while for $E_{M} \gg t_{1}, t_{2}$ only one process can happen. Concerning the coupling energy of MFs, we note that since $\mathbf{E}_{\mathbf{M}} \propto e^{-l / \xi_{0}}, E_{M}$ can be increased by decreasing the length of superconducting wire. For a wire whose length is about twice the coherence length of superconducting wire,
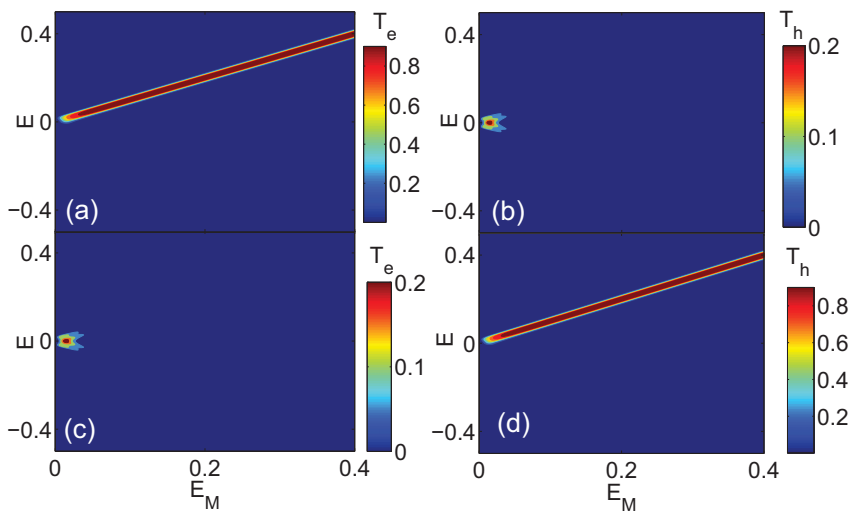

FIG. 4. (Color online) Contour plot of (a) $T_{e}$ and (b) $T_{h}$ as functions of MFs' coupling energy $E_{M}$ and incident energy $E$. We fixed the QD's energy level equal to $E_{M}: \varepsilon_{1}=\varepsilon_{2}=E_{M}$. In this case, only the ET process is allowed. In panels (c) and (d), contour plot of (c) $T_{e}$ and (d) $T_{h}$ as functions of MFs' coupling energy $E_{M}$ and incident energy $E$ are shown with the parameter $\varepsilon_{1}=-\varepsilon_{2}=E_{M}$. The CAR is allowed in this case. we have the coupling energy $E_{M} \sim 0.1 \Delta$ which is strong enough to distinguish the two nonlocal processes [38]. To examine the effect of temperature on the resonant CAR and ET, we note that the peak width of the resonant CAR or ET is approximately equal to $\left(\Gamma_{L}+\Gamma_{R}\right) / 2=0.03 \Delta$, while the temperature in the nowadays experiment can be easily maintained at $k_{B} T \sim 0.01 \Delta[15,39]$. Thus, the conductance peak would not be washed out by finite-temperature effects.

\section{NUMERICAL RESULTS FOR RASHBA SPIN-COUPLING SUPERCONDUCTING WIRE}

To further examine the correctness of our theory, we investigate the conductance of a superconducting wire with Rashba spin-orbit coupling using a tight-binding approach in this section. As shown in the following part, the results obtained agree very well with the effective model.

Tight-binding model. To model a one-dimensional $s$-wave superconductor with Rashba spin-orbit coupling, we use the following tight-binding model $[18,28]$ :

$$
\begin{aligned}
H_{q 1 \mathrm{D}}= & \sum_{\mathbf{i}, \mathbf{d}, \alpha}\left[-t\left(\psi_{\mathbf{i}+\mathbf{d}_{\mathbf{x}}, \alpha}^{\dagger} \psi_{i, \alpha}+\text { H.c. }\right)-\mu \psi_{\mathbf{i}, \alpha}^{\dagger} \psi_{\mathbf{i}, \alpha}\right] \\
& +\sum_{\mathbf{i}, \mathbf{d}_{\mathbf{x}}, \alpha, \beta}-i U_{R} \psi_{\mathbf{i}+\mathbf{d}_{\mathbf{x}}, \alpha}^{\dagger} \hat{z} \cdot\left(\vec{\sigma} \times \mathbf{d}_{\mathbf{x}}\right)_{\alpha \beta} \psi_{\mathbf{i}, \beta} \\
& +\sum_{\mathbf{i}, \alpha, \beta} \psi_{\mathbf{i}, \alpha}^{\dagger}\left[\left(V_{x} \sigma_{x}\right)_{\alpha \beta}+V_{\mathrm{imp}}(\mathbf{i}) \delta_{\alpha \beta}\right] \psi_{\mathbf{i}, \beta} \\
& +\sum_{\mathbf{i}, \alpha} \Delta \psi_{\mathbf{i}, \alpha}^{\dagger} \psi_{\mathbf{i},-\alpha}^{\dagger}+\text { H.c. }
\end{aligned}
$$

Here, $\mathbf{i}$ denotes the lattice sites, $\mathbf{d}_{\mathbf{x}}$ denotes the unit vectors which connect the nearest-neighbor sites in the $x$ directions. The spin indexes are denoted by $\alpha, \beta$ and $t$ denotes the hopping amplitude, $\mu$ is the chemical potential, $U_{R}$ is the Rashba coupling strength, $V_{x}$ is the Zeeman energy caused by a magnetic field along the wire in the $x$ direction. The pairing amplitude is denoted as $\Delta$ and $V_{\text {imp }}(\mathbf{i})$ is the onsite random impurity which is Gaussian distributed with variance $\overline{V_{\text {imp }}(\mathbf{i}) V_{\text {imp }}\left(\mathbf{i}^{\prime}\right)}=\omega^{2} \delta_{\mathbf{i}, \mathbf{i}^{\prime}}$.

Then, the wire is connected with the QDs. We consider a single-level QD with the spin included, the Hamiltonian of QDs is $H_{d}=\sum_{\mathbf{i}, \alpha} \varepsilon_{\mathbf{i}} \mathbf{d}_{\mathbf{i}, \alpha}^{\dagger} \mathbf{d}_{\mathbf{i}, \alpha}$ and the coupling between QDs and wires is $H_{c}=\sum_{\alpha}\left(t_{L} \mathbf{d}_{L, \alpha}^{\dagger} \psi_{N L, \alpha}+t_{R} \mathbf{d}_{R, \alpha}^{\dagger} \psi_{N R, \alpha}+\right.$ H.c. $)$. Here, $d_{L}$ and $d_{R}$ mean the annihilation operator of the left QD and right $\mathrm{QD}$, respectively. $N L$ and $N R$ mean the site position of the left end and right end of the TS wire.

As done in Ref. [18], we choose the parameters in the tightbinding model to reproduce experimental results. Here, $\Delta=$ $250 \mu \mathrm{eV}, t=25 \Delta$, and $U_{R}=2.5 \Delta$. We set $V_{x}=2 \Delta$ such that the superconducting wire can support MF end states by tuning the chemical potential. The length of the wire is $N_{x} a \approx$ $1 \mu \mathrm{m}$ which is about twice the superconducting coherence length $\xi_{0} \approx t a / \Delta$ and about half the length of the experimental value in Ref. [15].

Numerical results. Since the length of the wire is finite, the energy of the in-gap states versus the chemical potential exhibits oscillatory behavior in the topologically nontrivial region as shown in Fig. 5(a). This oscillation is due to the 

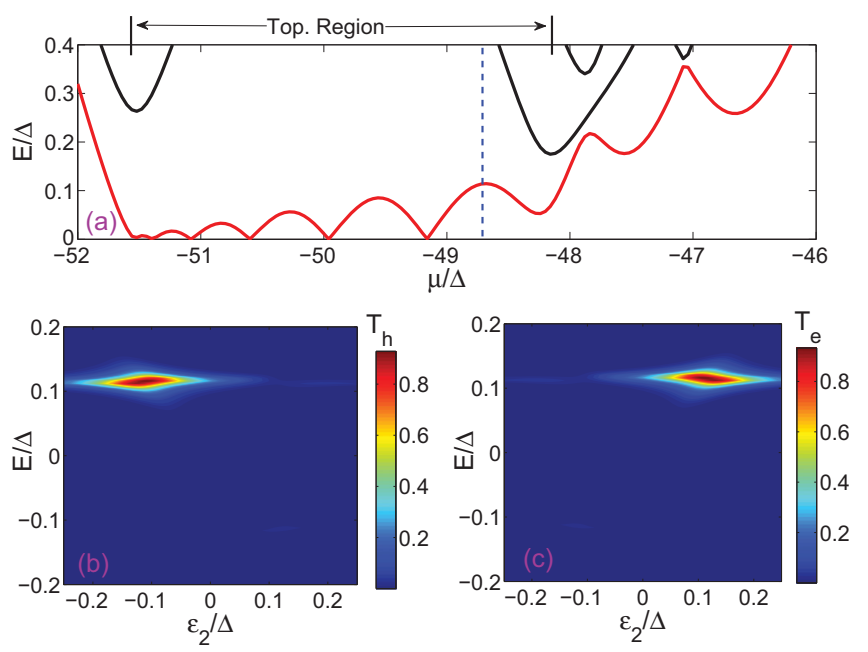

FIG. 5. (Color online) (a) The energy eigenvalues of a short 1D wire versus chemical potential, the lowest-energy states are indicated in red. The topological region is marked in the figure. (b) and (c) show the contour plot of $T_{h}$ and $T_{e}$, respectively, as functions of right QD's energy level $\varepsilon_{2}$ and incident energy $E$. The TS wire's chemical potential $\mu=-48.7$ is denoted by the dashed lines in (a). We fix the left QD's energy level to be $E_{M}: \varepsilon_{1}=E_{M}=0.11 \Delta$. We can see that the CAR is in resonant regime when $\varepsilon_{1}=-\varepsilon_{2}=E_{M}$, while the ET is in resonant regime when $\varepsilon_{1}=\varepsilon_{2}=E_{M}$.

fact that two MF end states can couple to each other with the coupling strength being an oscillating function of the chemical potential [24,33]. In the calculation, we fix the chemical potential $\mu=-48.7$ as denoted by the dashed lines in Fig. 5(a) so that the coupling strength is $E_{M}=0.11 \Delta$. We also fix the left QD's energy level with $\varepsilon_{1}=E_{M}=0.11 \Delta$ and tune the energy level of the right QD. As shown in Figs. 5(b) and 5(c), the results from the tight-binding model are in excellent agreement with that obtained from the effective model in Sec. III. Specifically, when $\varepsilon_{1}=-\varepsilon_{2}=E_{M}$, the CAR process is in the resonant regime, while for $\varepsilon_{1}=\varepsilon_{2}=E_{M}$, the ET process is in the resonant regime.

As shown in Fig. 5(a), the coupling strength of MFs oscillates as the chemical potential is varied. Therefore, it is very difficult to maintain the resonant condition $\varepsilon_{1}=-\varepsilon_{2}=E_{M}$ or $\varepsilon_{1}=\varepsilon_{2}=E_{M}$ in all regions. However, we will demonstrate below that even in the slightly off-resonant case, these three processes can still be clearly distinguished. In Fig. 2, we fix the QD's energy levels as $\varepsilon_{1}=-\varepsilon_{2}=0.08$ instead of resonant condition $\varepsilon_{1}=-\varepsilon_{2}=E_{M}$. Figures 6(a)-6(c) show the contour plot of $T_{A}, T_{e}$, and $T_{h}$ as functions of chemical potential $\mu$ and incident energy $E$, respectively. We can see that the CAR process is dominated in the whole region except at some point when $E_{M}$ equals to zero. Although it does show a resonant zero-bias peak (ZBP) when $E_{M}$ strictly equals to zero. However, the peak widths of ZBP are extremely small and would be washed out at finite temperatures. Similar to Fig. 6, we fix the QD's level with $\varepsilon_{1}=\varepsilon_{2}=0.08$ and tune the chemical potential of topological superconductor wire. As shown in Fig. 7, the ET is dominated in this situation. Thus, it is indeed possible to select different nonlocal transport processes by tuning the energy level of QDs.

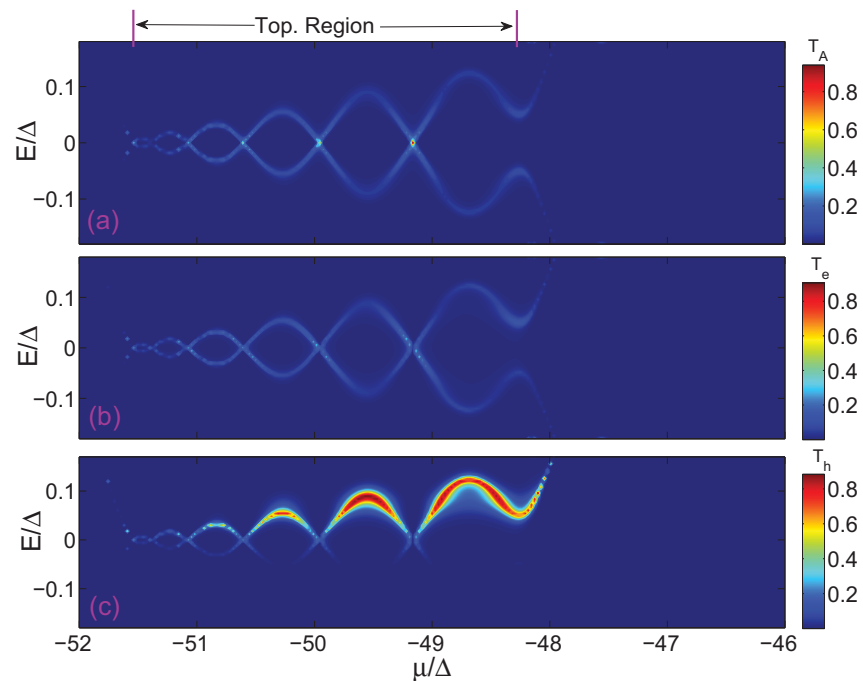

FIG. 6. (Color online) Contour plot of (a) $T_{A}$ and (b) $T_{e}$ and (c) $T_{h}$ as functions of chemical potential $\mu$ and incident energy $E$. We fixed the QD's energy level: $\varepsilon_{1}=-\varepsilon_{2}=0.08$. In this case, the CAR process is dominant.

Disorder effect. Finally, we examine the effect of disorder. Usually, disorder enhances the local Andreev reflection. For example, both nonmagnetic disorder and magnetic disorder can lead to ordinary fermionic Andreev bound states in TS wires [18]. However, these bound states can only be localized at one end of the superconducting wire. They can induce zero-bias peaks but certainly do not support nonlocal transport. In addition, weak antilocalization effect at an N-S junction can also induce zero-bias peaks. It is caused by the destructive interference effect between multiple scattering paths of electrons and holes near the N-S interface [20]. Since these processes happen at one end of TS wire, it only supports local Andreev reflection processes and has no effect



FIG. 7. (Color online) Contour plot of (a) $T_{A}$ and (b) $T_{e}$ and (c) $T_{h}$ as functions of chemical potential $\mu$ and incident energy $E$. We fixed the QD's energy level equal to $E_{M}: \varepsilon_{1}=\varepsilon_{2}=0.08$. The ET process is dominant in this case. 


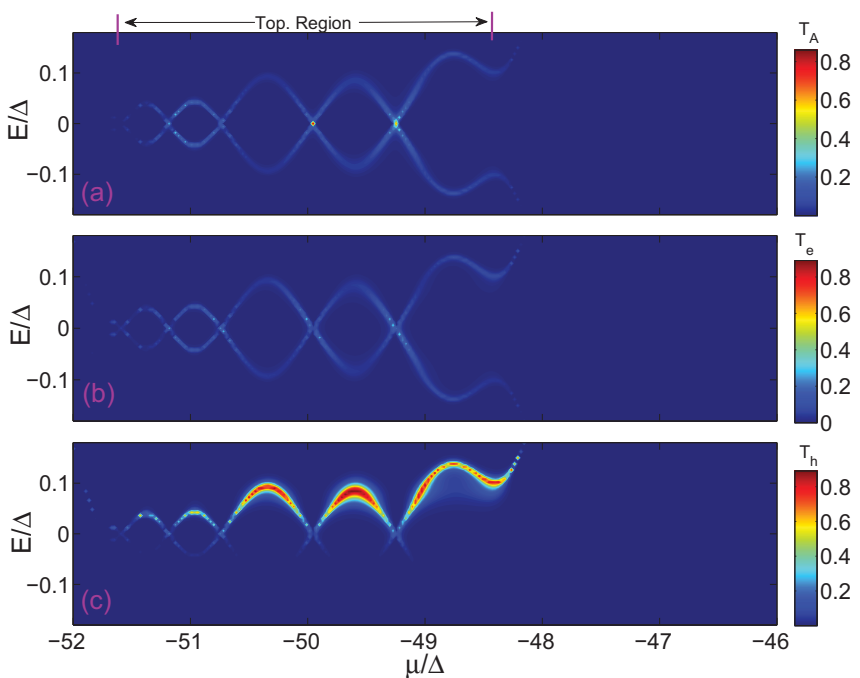

FIG. 8. (Color online) The disorder has little effect on the nonlocal process. Contour plot of (a) $T_{A}$ and (b) $T_{e}$ and (c) $T_{h}$ as functions of chemical potential $\mu$ and incident energy $E$. We fixed the QD's energy level equal to $E_{M}: \varepsilon_{1}=-\varepsilon_{2}=0.08$. We can see that the CAR process is still dominant despite the influence of disorder. The disorder strength is $\omega=\Delta$.

on nonlocal transport between two leads. Thus, in dealing with nonlocal transport, these local processes can be safely neglected. It is possible that two localized states localize at both ends of the wire that can support the nonlocal transport process. However, this is a very rare case. We have calculated transmission coefficients for local AR, CAR, and ET in the presence of a Gaussian disorder with disorder strength $\omega$ equal to the superconducting pairing strength. We found that we can clearly distinguish these three processes even in the presence of disorders. For instance, in Fig. 8, we have fixed the QD's energy level so that $\varepsilon_{1}=-\varepsilon_{2}=0.08$ is satisfied. From the figure, we see that the CAR process is still dominated in the topological region and is not affected by disorder.

\section{CONCLUSION}

With the aid of QDs, two different types of nonlocal electron transport processes via MFs in topological superconductor have been investigated. By adjusting energy levels of QDs, switching between these two processes can be achieved, i.e., the resonant ET process will happen when $\varepsilon_{1}=\varepsilon_{2}=E_{M}$, while for $\varepsilon_{1}=-\varepsilon_{2}=E_{M}$, the resonant CAR will occur. Since both ET and CAR manifest the nonlocal properties of MFs, they can be used to examine the nature of MFs. Importantly, all the conditions for observing resonant ET and CAR can be reached by present technology, and we expect that this nonlocal transport of MFs can be realized in the near future.

\section{ACKNOWLEDGMENTS}

We thank the insightful discussion with H. W. Liu, K. T. Law, and H. Jiang. We thank the support of HK RGC GRF Grants No. HKU707211 and No. AOE/P-04/08. J. Wang acknowledges the support of Grant No. HKU705611P.
[1] D. A. Ivanov, Phys. Rev. Lett. 86, 268 (2001).

[2] J. Alicea, Y. Oreg, G. Refael, F. von Oppen, and M. P. A. Fisher, Nat. Phys. 7, 412 (2011).

[3] A. Yu. Kitaev, Phys.-Usp. 44, 131 (2001).

[4] N. Read and D. Green, Phys. Rev. B 61, 10267 (2000).

[5] C. Nayak, S. H. Simon, A. Stern, M. Freedman, and S. Das Sarma, Rev. Mod. Phys. 80, 1083 (2008).

[6] J. D. Sau, R. M. Lutchyn, S. Tewari, and S. Das Sarma, Phys. Rev. Lett. 104, 040502 (2010).

[7] S. Fujimoto, Phys. Rev. B 77, 220501(R) (2008).

[8] M. Sato, Y. Takahashi, and S. Fujimoto, Phys. Rev. B 82, 134521 (2010).

[9] J. Alicea, Phys. Rev. B 81, 125318 (2010).

[10] R. M. Lutchyn, J. D. Sau, and S. Das Sarma, Phys. Rev. Lett. 105, 077001 (2010).

[11] Y. Oreg, G. Refael, and F. von Oppen, Phys. Rev. Lett. 105, 177002 (2010).

[12] A. C. Potter and P. A. Lee, Phys. Rev. B 83, 094525 (2011).

[13] K. T. Law, P. A. Lee, and T. K. Ng, Phys. Rev. Lett. 103, 237001 (2009).

[14] M. Wimmer, A. R. Akhmerov, J. P. Dahlhaus, and C. W. J. Beenakker, New J. Phys. 13, 053016 (2011).

[15] V. Mourik, K. Zuo, S. M. Frolov, S. R. Plissard, E. P. A. M. Bakkers, and L. P. Kouwenhoven, Science 336, 1003 (2012).

[16] M. T. Deng, C. L. Yu, G. Y. Huang, M. Larsson, P. Caroff, and H. Q. Xu, Nano Lett. 12, 6414 (2012).
[17] A. Das, Y. Ronen, Y. Most, Y. Oreg, M. Heiblum, and H. Shtrikman, Nat. Phys. 8, 887 (2012).

[18] J. Liu, A. C. Potter, K. T. Law, and P. A. Lee, Phys. Rev. Lett. 109, 267002 (2012).

[19] D. Bagrets and A. Altland, Phys. Rev. Lett. 109, 227005 (2012).

[20] D. I. Pikulin, J. P. Dahlhaus, M. Wimmer, H. Schomerus, and C. W. J. Beenakker, New J. Phys. 14, 125011 (2012).

[21] G. Kells, D. Meidan, and P. W. Brouwer, Phys. Rev. B 85, 060507(R) (2012).

[22] S. Tewari, T. D. Stanescu, J. D. Sau, and S. Das Sarma, Phys. Rev. B 86, 024504 (2012).

[23] F. Pientka, G. Kells, A. Romito, P. W. Brouwer, and F. von Oppen, Phys. Rev. Lett. 109, 227006 (2012).

[24] D. Rainis, L. Trifunovic, J. Klinovaja, and D. Loss, Phys. Rev. B 87, 024515 (2013).

[25] H. O. H. Churchill, V. Fatemi, K. Grove-Rasmussen, M. T. Deng, P. Caroff, H. Q. Xu, and C. M. Marcus, Phys. Rev. B 87, 241401(R) (2013).

[26] J. Nilsson, A. R. Akhmerov, and C. W. J. Beenakker, Phys. Rev. Lett. 101, 120403 (2008).

[27] Liang Fu, Phys. Rev. Lett. 104, 056402 (2010).

[28] Jie Liu, Fu-Chun Zhang, and K. T. Law, Phys. Rev. B 88, 064509 (2013).

[29] S. Tewari, C. Zhang, S. Das Sarma, C. Nayak, and D. H. Lee, Phys. Rev. Lett. 100, 027001 (2008). 
[30] Peiyue Wang, Yunshan Cao, Ming Gong, Gang Xiong, and Xin-Qi Li, Europhys. Lett. 103, 57016 (2013).

[31] H. F. Lu, H. Z. Lu, and S. Q. Shen, Phys. Rev. B 86, 075318 (2012).

[32] Björn Zocher and Bernd Rosenow, Phys. Rev. Lett. 111, 036802 (2013).

[33] S. Das Sarma, J. D. Sau, and T. D. Stanescu, Phys. Rev. B 86, 220506 (2012).

[34] P. A. Lee and D. S. Fisher, Phys. Rev. Lett. 47, 882 (1981); D. S. Fisher and P. A. Lee, Phys. Rev. B 23, 6851 (1981).
[35] Qing-feng Sun and X. C. Xie, J. Phys.: Condens. Matter 21, 344204 (2009).

[36] M. Leijnse and K. Flensberg, Phys. Rev. B 86, 134528 (2012).

[37] Silvano De Franceschi, Leo Kouwenhoven, Christian Schönenberger, and Wolfgang Wernsdorfer, Nat. Nanotechnol. 5, 703 (2010).

[38] Here we wish to emphasize that the length of wire should exceed the coherence length of superconducting wire, otherwise the CAR and ET via ordinary localized state may occur, which can blur the results.

[39] J. Schindele, A. Baumgartner, and C. Schönenberger, Phys. Rev. Lett. 109, 157002 (2012). 